PROCEEDINGS OF THE

AMERICAN MATHEMATICAL SOCIETY

Volume 138, Number 9, September 2010, Pages 3025-3035

S 0002-9939(10)10408-0

Article electronically published on April 27, 2010

\title{
ON THE PERIODICITY OF SOME FARHI ARITHMETICAL FUNCTIONS
}

QING-ZHONG JI AND CHUN-GANG JI

(Communicated by Wen-Ching Winnie Li)

\begin{abstract}
Let $k \in \mathbb{N}$. Let $f(x) \in \mathbb{Z}[x]$ be any polynomial such that $f(x)$ and $f(x+1) f(x+2) \cdots f(x+k)$ are coprime in $\mathbb{Q}[x]$. We call

$$
g_{k, f}(n):=\frac{|f(n) f(n+1) \cdots f(n+k)|}{\operatorname{lcm}(f(n), f(n+1), \cdots, f(n+k))}
$$

a Farhi arithmetic function. In this paper, we prove that $g_{k, f}$ is periodic. This generalizes the previous results of Farhi and Kane, and Hong and Yang.
\end{abstract}

\section{INTRODUCTION}

Throughout this paper, let $\mathbb{Q}, \mathbb{Z}$ and $\mathbb{N}$ denote the field of rational numbers, the ring of rational integers and the set of nonnegative integers. Let $\mathbb{N}^{*}=\mathbb{N} \backslash\{0\}$. As usual, let $v_{p}$ denote the normalized $p$-adic valuation of $\mathbb{Q}$, i.e., $v_{p}(a)=b$ if $p^{b} \| a$.

It is known that an equivalent variation of the Prime Number Theorem states that $\log \operatorname{lcm}(1,2, \cdots, n) \sim n$ as $n$ tends to infinity (see, e.g., [5]). One thus expects that a better understanding of the function $\operatorname{lcm}(1,2, \cdots, n)$ may entail a deeper understanding of the distribution of the prime numbers. Some progress has been made towards this direction. Before we state our main theorems, let us first give a short account on the recent results in this subject.

In his pioneering paper [2], Farhi introduced the following arithmetic functions:

$$
g_{k}(n):=\frac{n(n+1) \cdots(n+k)}{\operatorname{lcm}(n, n+1, \cdots, n+k)}, n \in \mathbb{N}^{*} .
$$

Farhi proved that the sequence $\left(g_{k}\right)_{k \in \mathbb{N}}$ satisfies the recursion relation:

$$
g_{k}(n)=\operatorname{gcd}\left(k !,(n+k) g_{k-1}(n)\right), \quad \forall n \in \mathbb{N}^{*} .
$$

Using this relation, Farhi proved

Theorem $1.1([2])$. The function $g_{k}(k \in \mathbb{N})$ is periodic and $k !$ is a period of $g_{k}$.

Received by the editors June 8, 2009.

2010 Mathematics Subject Classification. Primary 11A25; Secondary 11B83.

Key words and phrases. Arithmetical functions, least common multiple, periodicity.

The first author was partially supported by Grants No. 10571080 and 10871088 from the NNSF of China.

The second author was partially supported by Grants No. 10971098 and 10771103 from the NNSF of China.

(C)2010 American Mathematical Society Reverts to public domain 28 years from publication 
An interesting question is how to determine the least period of $g_{k}$ (see [2]). In [6], by using (11) and $g_{k}(1) \mid g_{k}(n)$ for any positive integer $n$, Hong and Yang gave a partial answer to this question. A complete solution to the question was given by Farhi and Kane in [3. They proved

Theorem 1.2 ([3], Theorem 3.2). The least period $T_{k}$ of $g_{k}$ is given by

$$
T_{k}=\prod_{p \text { prime }, p \leq k} p^{\delta_{p}(k)}
$$

where

$$
\delta_{p}(k)= \begin{cases}0, & \text { if } v_{p}(k+1) \geq \max _{1 \leq i \leq k}\left\{v_{p}(i)\right\}, \\ \max _{1 \leq i \leq k}\left\{v_{p}(i)\right\}, & \text { otherwise. }\end{cases}
$$

Let $g(n)$ be an arithmetic function defined on the set $\mathbb{Z} \backslash A$, where $A$ is a finite subset of $\mathbb{Z}$. If there exists an integer $T$ such that $g(n)=g(n+T)$ for all $n, n+T \in$ $\mathbb{Z} \backslash A$, then it is clear that the arithmetic function $g(n)$ can be extended to a periodic function defined on all the integers $\mathbb{Z}$.

Throughout this paper, let $k$ be a nonnegative integer and $f(x) \in \mathbb{Z}[x]$ such that

$$
\operatorname{gcd}(f(x), f(x+1) f(x+2) \cdots f(x+k))=1
$$

in $\mathbb{Q}[x]$. Set

$$
Z_{k, f}:=\{n \in \mathbb{Z} \mid f(n+i)=0 \text { for some } 0 \leq i \leq k\} .
$$

Then $Z_{k, f}$ is a finite subset of $\mathbb{Z}$. Set

$$
g_{k, f}(n)=\frac{|f(n) f(n+1) \cdots f(n+k)|}{\operatorname{lcm}(f(n), f(n+1), \cdots, f(n+k))},
$$

for $n \in \mathbb{Z} \backslash Z_{k, f}$. We call $g_{k, f}(n)$ a Farhi arithmetic function. In $\S 3$, we will prove

Theorem 1.3. Let $k$ be a nonnegative integer and $f(x) \in \mathbb{Z}[x]$ such that $\operatorname{gcd}(f(x)$, $f(x+1) f(x+2) \cdots f(x+k))=1$ in $\mathbb{Q}[x]$. Then the arithmetic function $g_{k, f}$ can be extended to a periodic arithmetic function defined on all the integers.

By assumption of $f(x)$ in Theorem 1.3, for any $1 \leq i \leq k$, there exist polynomials $a_{i}(x), b_{i}(x) \in \mathbb{Z}[x]$ and the smallest positive integer $C_{i}$ such that

$$
a_{i}(x) f(x)+b_{i}(x) f(x+i)=C_{i} .
$$

Let $C$ be the least common multiple of the $C_{i}$ 's, i.e.,

$$
C=\operatorname{lcm}\left(C_{1}, C_{2}, \cdots, C_{k}\right) .
$$

In the proof of Theorem 1.3, we will prove

Theorem 1.4. Let $T_{k, f}$ denote the least period of $g_{k, f}$. Then $T_{k, f} \mid C$.

Let $p$ be a prime. Define the arithmetic function $h_{k, f, p}$ by

$$
h_{k, f, p}(n):=v_{p}\left(g_{k, f}(n)\right) .
$$

If $p \nmid C$, using the definition of $g_{k, f}$, then we have $h_{k, f, p}(n)=0$ for any $n \in \mathbb{Z}$. If $p \mid C$, then $h_{k, f, p}$ is a periodic function by Theorem 1.3. Set

$$
S_{n}:=\{n, n+1, \cdots, n+k\}, n \in \mathbb{Z}
$$

and

$$
e_{p}:=\max \left\{v_{p}(\operatorname{gcd}(|f(n)|,|f(n+i)|)) \mid 1 \leq n \leq p^{v_{p}(C)}, 1 \leq i \leq k\right\} .
$$


In $\S 4$, we will prove

Theorem 1.5. For any prime $p$, let $T_{k, f, p}$ be the least period of the arithmetic function $h_{k, f, p}$. Then

(i) $p^{e_{p}}$ is a period of $h_{k, f, p}$ and $T_{k, f, p} \mid p^{e_{p}}$.

(ii) $T_{k, f, p}=1$ if and only if for any $1 \leq n \leq p^{e_{p}}$, we have

$$
v_{p}(\operatorname{gcd}(|f(n)|,|f(n+k+1)|)) \geq \max _{1 \leq i \leq k}\left\{v_{p}(f(n+i))\right\}
$$

or

$$
v_{p}(f(n))=v_{p}(f(n+k+1))<\max _{1 \leq i \leq k}\left\{v_{p}(f(n+i))\right\} .
$$

(iii) Let $1 \leq e \leq e_{p}$. Suppose that $p^{e}$ is a period of $h_{k, f, p}$. Then $T_{k, f, p}=p^{e}$ if and only if there exists an integer $n_{0}: 1 \leq n_{0} \leq p^{e}$ such that the following inequality holds:

$$
\begin{gathered}
\sum_{t=e}^{e_{p}} \max \left\{0, \#\left\{m \in S_{n_{0}}\left|p^{t}\right| f(m)\right\}-1\right\} \\
\neq \sum_{t=e}^{e_{p}} \max \left\{0, \#\left\{m \in S_{n_{0}}\left|p^{t}\right| f\left(m+p^{e-1}\right)\right\}-1\right\} .
\end{gathered}
$$

In particular, $T_{k, f, p}=p^{e_{p}}$ if and only if there exists an integer $n_{0}: 1 \leq n_{0} \leq p^{e_{p}}$ such that the following inequality holds:

$$
\#\left\{m \in S_{n_{0}}\left|p^{e_{p}}\right| f(m)\right\} \neq \#\left\{m \in S_{n_{0}}\left|p^{e_{p}}\right| f\left(m+p^{e_{p}-1}\right)\right\} .
$$

Remark. Let $T_{k, f, p}$ be the least period of $h_{k, f, p}$ for any prime $p$. Then

$$
T_{k, f}=\prod_{p} T_{k, f, p} .
$$

(This infinite product is meaningful, for almost all its terms are equal to 1.)

As an application of Theorem 1.5, in $\S 5$ we will give a new different proof of Theorem 3.2 of 3 .

Corollary 1.6. Let $k \in \mathbb{N}$ and $f(x)=x$. Then the least period $T_{k, f}$ of the Farhi arithmetic function $g_{k, f}$ is given by the formula (9).

Let $a, b \in \mathbb{Z}$ be any integer such that $\operatorname{gcd}(a, b)=1$ and $a>0$. Let $f(x)=a x+b$. By Theorem 1.3. we know that the Farhi arithmetic function

$$
g_{k, a x+b}(n)=\frac{|(a n+b)(a(n+1)+b) \cdots(a(n+k)+b)|}{\operatorname{lcm}(a n+b, a(n+1)+b, \cdots, a(n+k)+b)}
$$

can be extended to a periodic arithmetic function defined on all the integers. Now we define the arithmetical function $g_{k, a}$ by

$$
g_{k, a}(n)=\frac{|n(n+a) \cdots(n+k a)|}{\operatorname{lcm}(n, n+a, \cdots, n+k a)} .
$$

When $a=1$, the arithmetical function $g_{k, 1}$ is the arithmetical function $g_{k}$ defined by Farhi. It is clear that

$$
g_{k, a x+b}(n)=g_{k, a}(n a+b) .
$$

Hence the function $g_{k, a}$ can also be extended to a periodic arithmetic function defined on all the integers. In $\S 6$, we shall prove the following results: 
Theorem 1.7. Let $a, k$ be any two positive integers. Then the following assertions hold:

(i) The positive integer $a \cdot \operatorname{lcm}(1,2, \cdots, k)$ is a period of $g_{k, a}$.

(ii) A positive integer $S$ is a period of $g_{k, a}$ if and only if $S=a T$, where $T$ is a period of $g_{k}$.

(iii) Consequently, the least period $T_{k}(a)$ of $g_{k, a}$ is $a T_{k}(1)=a T_{k}$, where $T_{k}(1)=$ $T_{k}$ is the least period of $g_{k}$.

By (8) and Theorem 1.7, we have the following result:

Corollary 1.8. Let $a, k$ be any two positive integers and let $b \in \mathbb{Z}$ be any integer such that $\operatorname{gcd}(a, b)=1$. Then the least period $T_{k, a x+b}$ of the Farhi arithmetic function $g_{k, a x+b}$ is given by the following formula:

$$
T_{k, a x+b}=\prod_{p \text { prime, } p \leq k} p^{\delta_{p}(k)},
$$

where

$$
\delta_{p}(k)= \begin{cases}0, & \text { if } v_{p}(k+1) \geq \max _{1 \leq i \leq k}\left\{v_{p}(i)\right\} \text { or } p \mid a, \\ \max _{1 \leq i \leq k}\left\{v_{p}(i)\right\}, & \text { otherwise. }\end{cases}
$$

In $\S 7$, we will give some examples.

\section{TWO BASIC LEMMAS}

Lemma 2.1. Let $a_{1}, a_{2}, \cdots, a_{n}$ and $b_{1}, b_{2}, \cdots, b_{n}$ be any $2 n$ positive integers. If $\operatorname{gcd}\left(a_{i}, a_{j}\right)=\operatorname{gcd}\left(b_{i}, b_{j}\right)$ for any $1 \leq i<j \leq n$, then

$$
\frac{a_{1} a_{2} \cdots a_{n}}{\operatorname{lcm}\left(a_{1}, a_{2}, \cdots, a_{n}\right)}=\frac{b_{1} b_{2} \cdots b_{n}}{\operatorname{lcm}\left(b_{1}, b_{2}, \cdots, b_{n}\right)} .
$$

Proof. Let $p$ be any prime. It suffices to show that the following equality holds:

$$
v_{p}\left(\frac{a_{1} a_{2} \cdots a_{n}}{\operatorname{lcm}\left(a_{1}, a_{2}, \cdots, a_{n}\right)}\right)=v_{p}\left(\frac{b_{1} b_{2} \cdots b_{n}}{\operatorname{lcm}\left(b_{1}, b_{2}, \cdots, b_{n}\right)}\right),
$$

$$
\sum_{i=1}^{n} v_{p}\left(a_{i}\right)-\max _{1 \leq i \leq n}\left\{v_{p}\left(a_{i}\right)\right\}=\sum_{i=1}^{n} v_{p}\left(b_{i}\right)-\max _{1 \leq i \leq n}\left\{v_{p}\left(b_{i}\right)\right\} .
$$

By symmetry, it suffices to show that

$$
\sum_{i=1}^{n} v_{p}\left(a_{i}\right)-\max _{1 \leq i \leq n}\left\{v_{p}\left(a_{i}\right)\right\} \leq \sum_{i=1}^{n} v_{p}\left(b_{i}\right)-\max _{1 \leq i \leq n}\left\{v_{p}\left(b_{i}\right)\right\} .
$$

Without loss of generality, we assume that $v_{p}\left(a_{1}\right) \leq v_{p}\left(a_{2}\right) \leq \cdots \leq v_{p}\left(a_{n-1}\right) \leq$ $v_{p}\left(a_{n}\right)$. Then for any $1 \leq i \leq n-1$, we have

$$
v_{p}\left(a_{i}\right)=v_{p}\left(\operatorname{gcd}\left(a_{i}, a_{n}\right)\right)=v_{p}\left(\operatorname{gcd}\left(b_{i}, b_{n}\right)\right) \leq \min \left\{v_{p}\left(b_{i}\right), v_{p}\left(b_{n}\right)\right\} .
$$

Hence for any $1 \leq i \leq n-1$, we have $v_{p}\left(a_{i}\right) \leq v_{p}\left(b_{i}\right)$ and $v_{p}\left(a_{i}\right) \leq v_{p}\left(b_{n}\right)$. Let $v_{p}\left(b_{k}\right)=\max _{1 \leq i \leq n}\left\{v_{p}\left(b_{i}\right)\right\}$. Then

$$
\sum_{i=1}^{n-1} v_{p}\left(a_{i}\right) \leq v_{p}\left(b_{1}\right)+\cdots+v_{p}\left(b_{k-1}\right)+v_{p}\left(b_{n}\right)+v_{p}\left(b_{k+1}\right)+\cdots+v_{p}\left(b_{n-1}\right) .
$$

So (13) is true. This completes the proof of Lemma 2.1. 
Lemma 2.2. Let $k$ be a positive integer and $f(x) \in \mathbb{Z}[x]$ such that

$$
\operatorname{gcd}(f(x), f(x+1) f(x+2) \cdots f(x+k))=1
$$

in $\mathbb{Q}[x]$. Then $d_{i}(n)=\operatorname{gcd}(|f(n)|,|f(n+i)|)$ is periodic for any $1 \leq i \leq k$.

Proof. By assumption, for any $1 \leq i \leq k, f(x)$ and $f(x+i)$ are coprime in $\mathbb{Q}[x]$; hence there exist $a_{i}(x), b_{i}(x) \in \mathbb{Z}[x]$ and the smallest positive integer $C_{i}$ such that

$$
a_{i}(x) f(x)+b_{i}(x) f(x+i)=C_{i} .
$$

Hence for all $m \in \mathbb{Z}$, we have

$$
a_{i}(m) f(m)+b_{i}(m) f(m+i)=C_{i} .
$$

In the following, we will prove

$$
d_{i}(n)=d_{i}\left(n+C_{i}\right), \quad n \in \mathbb{Z} .
$$

Let $d_{i}=d_{i}(n)$ and $d_{i}^{\prime}=d_{i}\left(n+C_{i}\right)$. Then $d_{i} \mid f(n)$ and $d_{i} \mid f(n+i)$. By (15) , we have $d_{i} \mid C_{i}$. Hence, by the Taylor formula of $f$, we obtain that $d_{i} \mid f\left(n+C_{i}\right)$ and $d_{i} \mid f\left(n+i+C_{i}\right)$. Therefore $d_{i} \mid d_{i}^{\prime}$. Similarly, we have $d_{i}^{\prime} \mid d_{i}$. Hence $d_{i}=d_{i}^{\prime}$, i.e., (16) is true. This completes the proof of Lemma 2.2.

\section{The Proofs of Theorem 1.3 And Theorem 1.4}

Proof of Theorem 1.3. By the definition (3), $Z_{k, f}$ is a finite set and $g_{k, f}$ is well defined on the set $\mathbb{Z} \backslash Z_{k, f}$. First we prove that $g_{k, f}$ is periodic on the set $\mathbb{Z} \backslash Z_{k, f}$. For $1 \leq i \leq k$, by Lemma 2.2 $d_{i}(n)=\operatorname{gcd}(|f(n)|,|f(n+i)|)$ is periodic. Let $T_{i}$ be the least period of $d_{i}$. Then

$$
d_{i}(n)=d_{i}\left(n+T_{i}\right), \quad \text { for any } n \in \mathbb{Z} .
$$

Hence by the proof of Lemma 2.2, we have that $T_{i} \mid C_{i}$, where $C_{i}$ is defined by (14). Denote by $T$ (resp. $C$ ) the least common multiple of the $T_{i}$ 's (resp. $C_{i}$ 's), $i=1,2, \cdots, k$. Then $T \mid C$ and for any $1 \leq i \leq k$, we have

$$
d_{i}(n)=d_{i}(n+T) \quad \text { for } n \in \mathbb{Z} .
$$

Hence for any $0 \leq i<j \leq k$, we have

$$
d_{j-i}(n+i)=d_{j-i}(n+i+T) \quad \text { for } n \in \mathbb{Z},
$$

that is,

$$
\operatorname{gcd}(|f(n+i)|,|f(n+j)|)=\operatorname{gcd}(|f(n+i+T)|,|f(n+j+T)|) .
$$

So by Lemma 2.1 and the definition of $g_{k, f}$, we obtain that $g_{k, f}(n)=g_{k, f}(n+T)$ for any $n$ and $n+T \in \mathbb{Z} \backslash Z_{k, f}$. Hence $g_{k, f}(n)$ is periodic and $T$ is a period of $g_{k, f}$.

If $n \in Z_{k, f}$, then there exist a positive integer $a$ such that $n+a T \notin Z_{k, f}$. Hence the function $g_{k, f}$ can be extended to $g_{k, f}: \mathbb{Z} \longrightarrow \mathbb{Z}$, defined at $n \in Z_{k, f}$, by

$$
g_{k, f}(n)=g_{k, f}(n+a T) .
$$

This completes the proof of Theorem 1.3.

Proof of Theorem 1.4. It is obvious that the property $T \mid C$ implies that $C$ is a multiple of the least period $T_{k, f}$ of $g_{k, f}$. This completes the proof of Theorem 1.4. 


\section{The Proof of Theorem 1.5}

We use the same notation as in previous sections.

Proof. (i) By the definitions of $h_{k, f, p}$ and $g_{k, f}$, it suffices to show that $v_{p}\left(g_{k, f}(n)\right)=$ $v_{p}\left(g_{k, f}\left(n+p^{e_{p}}\right)\right)$ for any $n \in \mathbb{Z} \backslash Z_{k, f}$, i.e.,

$$
\begin{gathered}
\sum_{i=0}^{k} v_{p}(f(n+i))-\max _{0 \leq i \leq k}\left\{v_{p}(f(n+i))\right\} \\
=\sum_{i=0}^{k} v_{p}\left(f\left(n+i+p^{e_{p}}\right)\right)-\max _{0 \leq i \leq k}\left\{v_{p}\left(f\left(n+i+p^{e_{p}}\right)\right)\right\} .
\end{gathered}
$$

Let

$$
e_{i j}=v_{p}(\operatorname{gcd}(|f(n+i)|,|f(n+j)|))
$$

and

$$
e_{i j}^{\prime}=v_{p}\left(\operatorname{gcd}\left(\left|f\left(n+i+p^{e_{p}}\right)\right|,\left|f\left(n+j+p^{e_{p}}\right)\right|\right)\right)
$$

for any $0 \leq i<j \leq k$. By the proof of Lemma 2.1, it suffices to show that

$$
e_{i j}=e_{i j}^{\prime}
$$

By the assumption of $f(x)$, we have

$$
a_{j-i}(m) f(m)+b_{j-i}(m) f(m+j-i)=C_{j-i}, \quad m \in \mathbb{Z} .
$$

Let $m=n+i$. We have $p^{e_{i j}} \mid f(n+i)$ and $p^{e_{i j}} \mid f(n+j)$, so $p^{e_{i j}} \mid C_{j-i}$. Hence $e_{i j} \leq e_{p}$ by the definition of $e_{p}$. So $p^{e_{i j}}\left|f\left(n+i+p^{e_{p}}\right), p^{e_{i j}}\right| f\left(n+j+p^{e_{p}}\right)$. Therefore $e_{i j} \leq e_{i j}^{\prime}$. Similarly, we have $e_{i j}^{\prime} \leq e_{i j}$. Hence (17) is true. It is easy to see that $T_{k, f, p} \mid p^{e_{p}}$.

(ii) By (i) of Theorem 1.5, we know that $h_{k, f, p}$ is periodic and $p^{e_{p}}$ is a period. So $T_{k, f, p}=1$ if and only if $h_{k, f, p}(n)=h_{k, f, p}(n+1)$ for any $1 \leq n \leq p^{e_{p}}$. By the definition of $g_{k, f}$, we have $T_{k, f, p}=1$ if and only if for any $1 \leq n \leq p^{e_{p}}$,

$$
\begin{aligned}
& \sum_{i=0}^{k} v_{p}(f(n+i))-\max _{0 \leq i \leq k}\left\{v_{p}(f(n+i))\right\} \\
= & \sum_{i=1}^{k+1} v_{p}(f(n+i))-\max _{1 \leq i \leq k+1}\left\{v_{p}(f(n+i))\right\} .
\end{aligned}
$$

Hence $T_{k, f, p}=1$ if and only if for any $1 \leq n \leq p^{e_{p}}$,

$$
v_{p}(\operatorname{gcd}(|f(n)|,|f(n+k+1)|)) \geq \max _{1 \leq i \leq k}\left\{v_{p}(f(n+i))\right\}
$$

or

$$
v_{p}(f(n))=v_{p}(f(n+k+1))<\max _{1 \leq i \leq k}\left\{v_{p}(f(n+i))\right\} .
$$

(iii) Let $1 \leq e \leq e_{p}$. Suppose that $p^{e}$ is a period of $h_{k, f, p}$. Hence $p^{e}$ is the least period of $h_{k, f, p}$ if and only if $p^{e-1}$ is not a period of $h_{k, f, p}$. Therefore $p^{e}$ is the least period of $h_{k, f, p}$ if and only if there exists an integer $n_{0}: 1 \leq n_{0} \leq p^{e}$ such that the following inequality holds:

$$
h_{k, f, p}\left(n_{0}\right) \neq h_{k, f, p}\left(n_{0}+p^{e-1}\right) .
$$


By definition (5), we have

$$
\begin{aligned}
h_{k, f, p}\left(n_{0}\right) & =\sum_{i=0}^{k} v_{p}\left(f\left(n_{0}+i\right)\right)-\max _{0 \leq i \leq k}\left\{v_{p}\left(f\left(n_{0}+i\right)\right)\right\} \\
& =\sum_{t=1}^{\infty} \max \left\{0, \#\left\{m \in S_{n_{0}}\left|p^{t}\right| f(m)\right\}-1\right\}
\end{aligned}
$$

and

$$
h_{k, f, p}\left(n_{0}+p^{e-1}\right)=\sum_{t=1}^{\infty} \max \left\{0, \#\left\{m \in S_{n_{0}}\left|p^{t}\right| f\left(m+p^{e-1}\right)\right\}-1\right\} .
$$

Remark that the infinite sums of (18) and (19) are meaningful, for almost all their terms are equal to 0 . On the other hand, when $t \leq e-1$, we know that $p^{t} \mid f(m)$ if and only if $p^{t} \mid f\left(m+p^{e-1}\right)$. Hence by the definition of $e_{p}$, the inequality $h_{k, f, p}\left(n_{0}\right) \neq$ $h_{k, f, p}\left(n_{0}+p^{e-1}\right)$ holds if and only if the inequality

$$
\begin{gathered}
\sum_{t=e}^{e_{p}} \max \left\{0, \#\left\{m \in S_{n_{0}}\left|p^{t}\right| f(m)\right\}-1\right\} \\
\neq \sum_{t=e}^{e_{p}} \max \left\{0, \#\left\{m \in S_{n_{0}}\left|p^{t}\right| f\left(m+p^{e-1}\right)\right\}-1\right\}
\end{gathered}
$$

holds. In particular, $T_{k, f, p}=p^{e_{p}}$ if and only if there exists an integer $n_{0}: 1 \leq$ $n_{0} \leq p^{e_{p}}$ such that the following inequality holds:

$$
\#\left\{m \in S_{n_{0}}\left|p^{e_{p}}\right| f(m)\right\} \neq \#\left\{m \in S_{n_{0}}\left|p^{e_{p}}\right| f\left(m+p^{e_{p}-1}\right)\right\} .
$$

This completes the proof of Theorem 1.5

\section{The proof of Corollary 1.6}

Proof. When $k=0$, then $g_{0, f}=1$. Let $k \geq 1$. For $1 \leq i \leq k$, we have $C_{i}=i$ and $C=\operatorname{lcm}(1,2, \cdots, k)$. Hence we obtain

$$
T_{k, f}=\prod_{p \text { prime }, p \leq k} T_{k, f, p}
$$

Letting $p \leq k$ be a prime, it suffices to prove the following statements:

(I) $T_{k, f, p}=1$ if $v_{p}(k+1) \geq \max _{1 \leq i \leq k}\left\{v_{p}(i)\right\}$.

(II) $T_{k, f, p}=p^{v_{p}(C)}$ if $v_{p}(k+1)<\max _{1 \leq i \leq k}\left\{v_{p}(i)\right\}$.

We first prove (I). As $e_{p}=v_{p}(C)=\max _{1 \leq i \leq k}\left\{v_{p}(i)\right\}$, by assumption $v_{p}(k+1) \geq e_{p}$, we have $v_{p}(k+1)=e_{p}$ or $e_{p}+1$.

Case (a): $1 \leq n \leq p^{e_{p}}-1$; then $e=v_{p}(n)<e_{p}$. Hence $v_{p}(n)=v_{p}(n+k+1)$ and $n=p^{e} n_{1}, p \nmid n_{1}$. Set $i=p^{e} i_{0}, 1 \leq i_{0} \leq p-1$ such that $p \mid\left(n_{1}+i_{0}\right)$. Then $1 \leq i \leq k$ and $v_{p}(n+i)>v_{p}(n)$. Hence

$$
v_{p}(n)=v_{p}(n+k+1)<\max _{1 \leq i \leq k}\left\{v_{p}(n+i)\right\} .
$$

Case (b1): $n=p^{e_{p}}$ and $v_{p}(k+1)=e_{p}+1$. We have $k+1=p^{e_{p}+1}$. Let $i=p^{e_{p}}(p-1)$. Then $1 \leq i \leq k$ and $v_{p}(n+i)=e_{p}+1>e_{p}$. Hence

$$
v_{p}(n)=v_{p}(n+k+1)<\max _{1 \leq i \leq k}\left\{v_{p}(n+i)\right\} .
$$


Case (b2): $n=p^{e_{p}}$ and $v_{p}(k+1)=e_{p}$. We have $k+1=p^{e_{p}} u$, where $2 \leq u \leq p-1$. Hence $k=u p^{e_{p}}-1$. If $i>0$ and $v_{p}(n+i)>e_{p}$, then $i \geq p^{e_{p}}(p-1)>k$. Hence $\max _{1 \leq i \leq k}\left\{v_{p}(n+i)\right\} \leq e_{p}$. Therefore

$$
v_{p}(\operatorname{gcd}(|n|,|n+k+1|)) \geq \max _{1 \leq i \leq k}\left\{v_{p}(n+i)\right\} .
$$

By (20), (21), (22) and using (ii) of Theorem 1.5, we have $T_{k, f, p}=1$.

(II) Note that $e_{p}=v_{p}(C)$. Hence

$$
k=a_{0}+a_{1} p+\cdots+a_{e_{p}} p^{e_{p}}, \quad 0 \leq a_{i} \leq p-1, \quad i=0,1, \cdots, e_{p}, a_{e_{p}} \neq 0 .
$$

It is easy to show that the inequality $v_{p}(k+1) \geq e_{p}$ holds if and only if $a_{0}=a_{1}=$ $\cdots=a_{e_{p}-1}=p-1$.

Assume that the inequality $v_{p}(k+1)<e_{p}=v_{p}(C)=\max _{1 \leq i \leq k} v_{p}(i)$ holds. Then there exists an integer $r: 0 \leq r \leq e_{p}-1$ such that the following conditions hold:

$$
0 \leq a_{r} \leq p-2 \text { and } a_{r+1}=\cdots=a_{e_{p}-1}=p-1 .
$$

Set

$$
n_{0}= \begin{cases}p^{e_{p}}, & \text { if } r=e_{p}-1 \\ \left(p-1-a_{r}\right) p^{r}, & \text { if } 0 \leq r \leq e_{p}-2 .\end{cases}
$$

Then we have

$$
\#\left\{m \in S_{n_{0}}\left|p^{e_{p}}\right| m\right\}= \begin{cases}a_{e_{p}}+1, & \text { if } r=e_{p}-1 \\ a_{e_{p}}, & \text { if } 0 \leq r \leq e_{p}-2\end{cases}
$$

and

$$
\#\left\{m \in S_{n_{0}}\left|p^{e_{p}}\right|\left(m+p^{e_{p}-1}\right)\right\}= \begin{cases}a_{e_{p}}, & \text { if } r=e_{p}-1, \\ a_{e_{p}}+1, & \text { if } 0 \leq r \leq e_{p}-2 .\end{cases}
$$

By (iii) of Theorem 1.5. we know that $p^{e_{p}}=p^{v_{p}(C)}$ is the least period of $h_{k, f, p}$. This completes the proof of Corollary 1.6.

\section{The Proof of Theorem 1.7}

Proof. (i) Set $S=a \cdot \operatorname{lcm}(1,2, \cdots, k)$. Let $n$ be any positive integer. For any $0 \leq i<j \leq k$, it is clear that $\operatorname{gcd}(n+i a, n+j a)=\operatorname{gcd}(n+S+i a, n+S+j a)$. Hence $g_{k, a}(n+S)=g_{k, a}(n)$ follows from Lemma 2.1 .

(ii) Suppose $S$ is a period of $g_{k, a}$. Then $g_{k, a}(n)=g_{k, a}(n+S)$ for all $n \in \mathbb{N}^{*}$. In particular, we have $g_{k, a}(n a)=g_{k, a}(n a+S)$. Since

$$
g_{k, a}(n a)=\frac{n a \cdot(n a+a) \cdots(n a+k a)}{\operatorname{lcm}(n a, n a+a, \cdots, n a+k a)}=\frac{n(n+1) \cdots(n+k)}{\operatorname{lcm}(n, n+1, \cdots, n+k)} \cdot a^{k}
$$

and

$$
g_{k, a}(n a+S)=\frac{(n a+S)(n a+a+S) \cdots(n a+k a+S)}{\operatorname{lcm}(n a+S, n a+a+S, \cdots, n a+k a+S)},
$$

we have

$$
g_{k}(n) \cdot a^{k}=g_{k, a}(n a)=g_{k, a}(n a+S)
$$

and

$$
g_{k}(n) \cdot a^{k}=\frac{(n a+S)(n a+a+S) \cdots(n a+k a+S)}{\operatorname{lcm}(n a+S, n a+a+S, \cdots, n a+k a+S)} .
$$


We claim that $a \mid S$. Let $\operatorname{gcd}(a, S)=d, a=a_{1} d, S=S_{1} d$. Then $\operatorname{gcd}\left(a_{1}, S_{1}\right)=1$. By using (24), we have

$$
a_{1}^{k} \mid\left(n a_{1}+S_{1}\right)\left(n a_{1}+a_{1}+S_{1}\right) \cdots\left(n a_{1}+k a_{1}+S_{1}\right) .
$$

Because $\operatorname{gcd}\left(a_{1}, n a_{1}+i a_{1}+S_{1}\right)=1$ for any $0 \leq i \leq k$, we have $a_{1}=1$. Hence $a \mid S$. Let $S=a T$. Then using (23), we have

$$
g_{k}(n) \cdot a^{k}=g_{k, a}(n a)=g_{k, a}(n a+a T)=g_{k}(n+T) \cdot a^{k} .
$$

Hence $g_{k}(n+T)=g_{k}(n)$ for all $n \in \mathbb{N}^{*}$; i.e., $T$ is a period of $g_{k}(n)$.

Conversely, suppose $T$ is a period of $g_{k}(n)$. Let $n$ be any positive integer. If $d=\operatorname{gcd}(n, a), n=n_{1} d, a=a_{1} d$, then $\operatorname{gcd}\left(n_{1}, a_{1}\right)=1$ and

$$
g_{k, a}(n)=g_{k, a_{1}}\left(n_{1}\right) \cdot d^{k}, \quad g_{k, a}(n+a T)=g_{k, a_{1}}\left(n_{1}+a_{1} T\right) \cdot d^{k} .
$$

Hence, without loss of generality, we assume that $(n, a)=1$. Therefore

$$
\operatorname{gcd}\left(a, g_{k, a}(n)\right)=1, \quad \operatorname{gcd}\left(a, g_{k, a}(n+a T)\right)=1 .
$$

Hence by using (25), we have

$$
g_{k, a}(n)=g_{k, a}(n+a T)
$$

if and only if

$$
v_{p}\left(g_{k, a}(n)\right)=v_{p}\left(g_{k, a}(n+a T)\right)
$$

for any prime $p \nmid a$.

Let $p$ be any prime such that $p \nmid a$ and let $N$ be a positive integer greater than $v_{p}(k !)$. Then there exists a unique positive integer $m$ such that $1 \leq m<p^{N}$ and

$$
m a \equiv 1\left(\bmod p^{N}\right) .
$$

Let $p, n, a, m$ be as above and $0 \leq i<j \leq k$. Then for any integer $l$, there are

$$
v_{p}(\operatorname{gcd}(n+a l+a i,(j-i) a))=v_{p}(\operatorname{gcd}(m n+l+i, j-i)) .
$$

Let

$$
\operatorname{gcd}(n+a l+a i,(j-i) a)=p^{x_{i j}} w, \quad p \nmid w
$$

and

$$
\operatorname{gcd}(m n+l+i, j-i)=p^{y_{i j}} u, \quad p \nmid u .
$$

Then by (29), there exists $s_{1}, t_{1} \in \mathbb{Z}$ such that $(n+a l+a i) s_{1}+(j-i) a t_{1}=p^{x_{i j}} w$. Multiplying by $m$ on both sides, we have $(m n+a m l+a m i) s_{1}+(j-i) m a t_{1}=p^{x_{i j}} m w$. Using (27), we have $(m n+l+i) s_{1}+(j-i) t_{1}=p^{x_{i j}} m w-p^{N} \delta$. By (30), we have $y_{i j} \leq$ $x_{i j}$. Conversely, by (30), there exist $s_{2}, t_{2} \in \mathbb{Z}$ such that $(m n+l+i) s_{2}+(j-i) t_{2}=$ $p^{y_{i j}} u$. Multiplying by $a$ on both sides, we have $(m n a+a l+a i) s_{2}+(j-i) a t_{2}=p^{y_{i j}} a u$. Similarly, we have $x_{i j} \leq y_{i j}$. So $x_{i j}=y_{i j}$ and (28) is true. Let $l=0$ and $T$. By using (28) we have

$$
v_{p}(\operatorname{gcd}(n+a i, n+a j))=v_{p}(\operatorname{gcd}(m n+i, m n+j))
$$

and

$$
v_{p}(\operatorname{gcd}(n+a T+a i, n+a T+a j))=v_{p}(\operatorname{gcd}(m n+T+i, m n+T+j))
$$

for any $0 \leq i<j \leq k$. By the proof of Lemma 2.1, we have

$$
v_{p}\left(g_{k, a}(n)\right)=v_{p}\left(g_{k}(m n)\right), \quad v_{p}\left(g_{k, a}(n+a T)\right)=v_{p}\left(g_{k}(m n+T)\right) .
$$


TABLE 1

The least period $T_{k, f}$ of $g_{k, f}$ with $f(x)=x^{2}+b$

\begin{tabular}{|c|c|c|c|c|c|c|}
\hline$f(x)$ & 1 & 2 & 3 & 4 & 5 & 6 \\
\hline$x^{2}+1$ & 5 & $2 \cdot 5$ & $2 \cdot 3 \cdot 5 \cdot 13$ & $2 \cdot 3 \cdot 5 \cdot 13$ & $2 \cdot 3 \cdot 5 \cdot 13 \cdot 29$ & $2 \cdot 3 \cdot 5 \cdot 13 \cdot 29$ \\
\hline$x^{2}+2$ & $3^{2}$ & $2 \cdot 3^{2}$ & $2 \cdot 3^{2} \cdot 17$ & $2 \cdot 3^{2} \cdot 17$ & $2 \cdot 3^{2} \cdot 5 \cdot 11 \cdot 17$ & $2 \cdot 3^{2} \cdot 5 \cdot 11 \cdot 17$ \\
\hline$x^{2}+3$ & 13 & $2 \cdot 13$ & $2 \cdot 3 \cdot 7 \cdot 13$ & $2 \cdot 3 \cdot 7 \cdot 13$ & $2 \cdot 3 \cdot 5 \cdot 7 \cdot 13 \cdot 37$ & $2 \cdot 3 \cdot 5 \cdot 7 \cdot 13 \cdot 37$ \\
\hline$x^{2}+4$ & 17 & $2 \cdot 5 \cdot 17$ & $2 \cdot 3 \cdot 5^{2} \cdot 17$ & $2^{2} \cdot 3 \cdot 5^{2} \cdot 17$ & $2^{2} \cdot 3 \cdot 5^{2} \cdot 17 \cdot 41$ & $2^{2} \cdot 3 \cdot 5^{2} \cdot 13 \cdot 17 \cdot 41$ \\
\hline$x^{2}+5$ & $3 \cdot 7$ & $2 \cdot 3 \cdot 7$ & $2 \cdot 3 \cdot 7 \cdot 29$ & $2 \cdot 3^{2} \cdot 7 \cdot 29$ & $2 \cdot 3^{2} \cdot 5 \cdot 7 \cdot 29$ & $2 \cdot 3^{2} \cdot 5 \cdot 7 \cdot 29$ \\
\hline$x^{2}+6$ & $5^{2}$ & $2 \cdot 5^{2} \cdot 7$ & $2 \cdot 3 \cdot 5^{2} \cdot 7 \cdot 11$ & $2 \cdot 3 \cdot 5^{2} \cdot 7 \cdot 11$ & $2 \cdot 3 \cdot 5^{2} \cdot 7^{2} \cdot 11$ & $2 \cdot 3 \cdot 5^{2} \cdot 7^{2} \cdot 11$ \\
\hline
\end{tabular}

So using $g_{k}(m n)=g_{k}(m n+T)$, we have $v_{p}\left(g_{k, a}(n+a T)\right)=v_{p}\left(g_{k, a}(n)\right)$ for any prime $p$ such that $p \nmid a$. Hence $g_{k, a}(n+a T)=g_{k, a}(n)$ and $a T$ is a period of $g_{k, a}(n)$.

The proof of (iii) follows from (ii). This completes the proof of Theorem 1.7 .

Proof of Corollary 1.8, (i) Assume that $p \mid a$. Then it is clear that the equality $v_{p}\left(g_{k, a x+b}(n)\right)=0$ holds for any integer $n$ when $g_{k, a x+b}(n)$ is well defined.

(ii) Assume that $p$ is not a prime factor of $a$. By the formula (8), we have that $T_{p}$ is a period of $v_{p}\left(g_{k, a x+b}(n)\right)$ if and only if $a T_{p}$ is a period of $v_{p}\left(g_{k, a}(n)\right)$. Hence, by Theorem 1.7, we have that $T_{p}$ is a period of $v_{p}\left(g_{k, a x+b}(n)\right)$ if and only if $T_{p}$ is a period of $v_{p}\left(g_{k}(n)\right)$. Therefore Corollary 1.8 is obtained by Theorem 1.2 .

\section{EXAMPLES}

Lemma 7.1. Let $f_{1}(x)=f_{2}(x)^{r}$, where $r \geq 1$ is an integer. Then $T_{k, f_{1}}=T_{k, f_{2}}$.

Proof. By (4), we have $g_{k, f_{1}}(n)=g_{k, f_{2}}(n)^{r}$. Hence the result is obvious.

Example. Let $f(x)=x^{r}, r \geq 1$. Then by Lemma 7.1] we have $T_{k, x^{r}}=T_{k, x}$, where $T_{k, x}$ is given by the formula (9).

Example. Let $f(x)=x^{2}+b$. For $1 \leq i \leq k$, we have

$$
\begin{gathered}
(2 x+3 i)\left(x^{2}+b\right)+(-2 x+i)\left((x+i)^{2}+b\right)=i\left(i^{2}+4 b\right), \text { if } i \text { is odd, } \\
(x+3 j)\left(x^{2}+b\right)+(-x+j)\left((x+2 j)^{2}+b\right)=4 j\left(j^{2}+b\right), \text { if } i=2 j .
\end{gathered}
$$

Hence

$$
C_{i}= \begin{cases}i\left(i^{2}+4 b\right), & \text { if } i \text { is odd }, \\ 4 j\left(j^{2}+b\right), & \text { if } i=2 j .\end{cases}
$$

Hence, given any $k \in \mathbb{N}$ and $b \in \mathbb{Z}$, by Theorem 1.5, we can determine the least period $T_{k, f}$ of the arithmetic function $g_{k, f}$. For $1 \leq k \leq 6$ and $1 \leq b \leq 6$, Table 1 gives the $T_{k, f}$ 's.

Example. Let $f(x)=x^{3}+b$. For $1 \leq i \leq k$, we have

$$
a_{i}(x)\left(x^{3}+b\right)+b_{i}(x)\left((x+i)^{3}+b\right)=C_{i},
$$

where

$$
a_{i}(x)= \begin{cases}6 i^{2} x^{2}+\left(15 i^{3}-9\right) x+10 i^{4}-18 i, & \text { if } 3 \nmid i ; \\ 6 j^{2} x^{2}+\left(45 j^{3}-1\right) x+90 i^{4}-6 i, & \text { if } i=3 j .\end{cases}
$$


TABLE 2

The least period $T_{k, f}$ of $g_{k, f}$ with $f(x)=x^{3}+b$

\begin{tabular}{|c|c|c|c|c|c|c|}
\hline$f(x)$ & 1 & 2 & 3 & 4 & 5 & 6 \\
\hline$x^{3}+1$ & $2 \cdot 7$ & $2 \cdot 7 \cdot 13$ & $2 \cdot 3 \cdot 7 \cdot 13$ & $2^{2} \cdot 3 \cdot 7 \cdot 11 \cdot 13 \cdot 17 \cdot 31$ & $2^{2} \cdot 3 \cdot 5 \cdot 7 \cdot 13 \cdot 17 \cdot 31 \cdot 43$ & $2^{2} \cdot 3 \cdot 5 \cdot 7 \cdot 13 \cdot 17 \cdot 19 \cdot 31 \cdot 43$ \\
\hline$x^{3}+2$ & $2 \cdot 7$ & $2 \cdot 7 \cdot 13$ & $2 \cdot 3 \cdot 7 \cdot 13$ & $2 \cdot 3 \cdot 7 \cdot 11 \cdot 13 \cdot 17 \cdot 31$ & $2 \cdot 3 \cdot 5 \cdot 7 \cdot 13 \cdot 17 \cdot 31 \cdot 43$ & $2 \cdot 3 \cdot 5 \cdot 7 \cdot 13 \cdot 17 \cdot 19 \cdot 31 \cdot 43$ \\
\hline$x^{3}+3$ & $2 \cdot 7$ & $2 \cdot 7 \cdot 13$ & $2 \cdot 3 \cdot 7 \cdot 13$ & $2^{2} \cdot 3 \cdot 7 \cdot 11 \cdot 13 \cdot 17 \cdot 31$ & $2^{2} \cdot 3 \cdot 5 \cdot 7 \cdot 13 \cdot 17 \cdot 31 \cdot 43$ & $2^{2} \cdot 3 \cdot 5 \cdot 7 \cdot 13 \cdot 17 \cdot 19 \cdot 31 \cdot 43$ \\
\hline$x^{3}+4$ & $2 \cdot 7$ & $2 \cdot 7 \cdot 13$ & $2 \cdot 3 \cdot 7 \cdot 13$ & $2 \cdot 3 \cdot 7 \cdot 11 \cdot 13 \cdot 17 \cdot 31$ & $2 \cdot 3 \cdot 5 \cdot 7 \cdot 13 \cdot 17 \cdot 31 \cdot 43$ & $2 \cdot 3 \cdot 5 \cdot 7 \cdot 13 \cdot 17 \cdot 19 \cdot 31 \cdot 43$ \\
\hline$x^{3}+5$ & $2 \cdot 7$ & $2 \cdot 7 \cdot 13$ & $2 \cdot 3 \cdot 7 \cdot 13$ & $2^{2} \cdot 3 \cdot 7 \cdot 11 \cdot 13 \cdot 17 \cdot 31$ & $2^{2} \cdot 3 \cdot 5 \cdot 7 \cdot 13 \cdot 17 \cdot 31 \cdot 43$ & $2^{2} \cdot 3 \cdot 5 \cdot 7 \cdot 13 \cdot 17 \cdot 19 \cdot 31 \cdot 43$ \\
\hline$x^{3}+6$ & $2 \cdot 7$ & $2 \cdot 7 \cdot 13$ & $2 \cdot 3 \cdot 7 \cdot 13$ & $2 \cdot 3 \cdot 7 \cdot 11 \cdot 13 \cdot 17 \cdot 31$ & $2 \cdot 3 \cdot 5 \cdot 7 \cdot 13 \cdot 17 \cdot 31 \cdot 43$ & $2 \cdot 3 \cdot 5 \cdot 7 \cdot 13 \cdot 17 \cdot 19 \cdot 31 \cdot 43$ \\
\hline
\end{tabular}

$$
\begin{gathered}
b_{i}(x)= \begin{cases}-6 i^{2} x^{2}+\left(3 i^{3}+9\right) x-i^{4}-9 i, & \text { if } 3 \nmid i ; \\
-6 j^{2} x^{2}+\left(9 j^{3}+1\right) x-9 j^{4}-3 j, & \text { if } i=3 j .\end{cases} \\
C_{i}= \begin{cases}-i^{7}-27 i, & \text { if } 3 \nmid i ; \\
-3^{5} j^{7}-9 j, & \text { if } i=3 j .\end{cases}
\end{gathered}
$$

Hence, given any $k \in \mathbb{N}$ and $b \in \mathbb{Z}$, by Theorem 1.5, we can determine the least period $T_{k, f}$ of the arithmetic function $g_{k, f}$. For $1 \leq k \leq 6$ and $1 \leq b \leq 6$, Table 2 gives the $T_{k, f}$ 's.

\section{REFERENCES}

[1] B. Farhi, Minorations non triviales du plus petit commun multiple de certaines suites finies d'entiers C. R. Math. Acad. Sci. Paris, 341(2005), 469-474. MR2180812 (2006g:11006)

[2] B. Farhi, Nontrivial lower bounds for the least common multiple of some finite sequences of integers, J. Number Theory, 125(2007), 393-411. MR2332595(2008i:11001)

[3] B. Farhi and D. Kane, New results on the least common multiple of consecutive integers, Proc. Amer. Math. Soc., 137(2009), 1933-1939. MR2480273(2010a:11004)

[4] B. Green and T. Tao, The primes contain arbitrarily long arithmetic progressions, Ann. of Math. (2) 167(2008), 481-548. MR2415379 (2009e:11181)

[5] G. H. Hardy and E. M. Wright, Theory of Numbers, fifth ed., Oxford Univ. Press, London, 1979. MR.568909 (81i:10002)

[6] S. Hong and Y. Yang, On the periodicity of an arithmetical function, C. R. Math. Acad. Sci. Paris, 346(2008), 717-721. MR2427068(2009e:11007)

Department of Mathematics, Nanjing University, Nanjing 210093, People's Republic OF CHINA

E-mail address: qingzhji@nju.edu.cn

Department of Mathematics, Nanjing Normal University, Nanjing 210097, People's Republic of China

E-mail address: cgji@njnu.edu.cn 